\title{
ROBOT AS PARTNER: VEHICLE TELEOPERATION WITH COLLABORATIVE CONTROL
}

Terrence Fong and Charles Thorpe

The Robotics Institute, Carnegie Mellon University, Pittsburgh, Pennsylvania

Charles Baur

Swiss Federal Institute of Technology, Lausanne, Switzerland

\begin{abstract}
We have developed a new teleoperation system model called collaborative control. With this model, the robot asks the human questions, to obtain assistance with cognition and perception during task execution. This enables the human to support the robot and to compensate for inadequacies in autonomy. In the following, we review the system models conventionally used in teleoperation, describe collaborative control, and discuss its use.
\end{abstract}

Keywords: Collaborative control, human-robot interaction, vehicle teleoperation.

\section{Introduction}

In teleoperation, a robot is commonly viewed as a tool: a device capable of performing tasks on command. As such, a robot has limited freedom to act and will always perform poorly whenever its capabilities are ill-suited for the task at hand. This is particularly true when high-level perceptual functions (e.g., object recognition) are involved. Moreover, even if a robot realizes that it is performing poorly, it usually has no way to ask for (or to gain) assistance.

The problem is that the "robot as tool" paradigm is extremely limiting, i.e., it restricts the human-robot relationship to that of master-slave. As a result, the system's capability is strictly bound to the operator's skill and the quality of the user interface. In order to make teleoperation better performing, therefore, we must find a new approach. What we need is a paradigm that is more flexible, that encourages human-robot synergy, and that allows robots to work as partners (if not as peers). 


\section{Collaborative Control}

\subsection{A Robot-Centric System Model}

To address this need, we have developed collaborative control, a system model in which human and robot work together[4, 5]. Instead of a supervisor dictating to a subordinate, the human and the robot engage in dialogue to exchange information, to ask questions, and to resolve differences. With this approach, human-robot interaction is more natural, more balanced, and more direct than conventional approaches.

With collaborative control, the human functions as a resource for the robot, providing information and processing just like other system modules. In particular, the robot is allowed to ask the human questions as it works, to obtain assistance with perception and cognition. This allows the human to compensate for limitations of autonomy. Moreover, since the robot is aware that the human may not respond, collaborative control enables dynamic, fine-grained sharing of control. With other forms of teleoperation, the division of labor is pre-defined or is coarsely switched on a per-task basis.

To understand how this works in practice, consider the following situation: a mobile robot is driving forward when it has difficulty deciding if there is an obstacle in its way (e.g., range sensors return conflicting information). At this point, the robot must make a decision. With conventional design, there are three choices: wait for the path to become clear, look for a way around, or ignore the conflict and continue forward. All of these strategies have significant problems: "wait until clear" may cause indefinite delay; "make a detour" may consume excessive resources; and "drive through" may result in excessive damage.

With collaborative control, the robot can ask for human assistance. In this case, the robot can present the sensor data (e.g., a camera image) to the human and ask his opinion. Once the human sees the data, he may decide (based on experience or interpretation) that "drive through" is acceptable. In other words, through collaboration, the robot can avoid needless delay and having to make an unnecessary detour.

Collaborative control provides an effective framework for coordination. Since the human can only attend to one module (or robot) at a time, we arbitrate among the requests to select which is presented. This allows human attention to be directed where it is most needed, in terms of safety, priority, etc. Additionally, because collaborative control incorporates a user model for dialogue management, it can accommodate users with varied backgrounds and capabilities. Thus, the robot can ask questions and can interpret responses, based on the user's expertise, preferences, and other characteristics. 


\section{Related Research}

Some robot control architectures have addressed the problem of mixing humans with robots. One method is to incorporate humans as a system module[8]. Another method is prioritized control, the classic example of which is NASREM[1]. In both these approaches, the human only provides command input. With collaborative control, however, the human contributes his expertise wherever it can be used.

Teleassistance tries to improve teleoperation by supplying aid to the operator in the same manner that an expert would[7]. Collaborative control takes the opposite approach: it provides the robot with human assistance. Although adjustable autonomy (e.g., [3]) shares some aspects of collaborative control, human-robot dialogue is not used as a mechanism for adaptation and coordination.

In many ways, collaborative control is most similar to remote expert systems. For example, Bauer et al. describe a wearable computer with a camera that enables office-based experts to see what a field technician sees[2]. The parallel with collaborative control is clear: the robot is like the field technician (i.e., it is skilled, but may need help) and the operator is like the expert (i.e., he can provide assistance when needed).

\section{Conventional System Models}

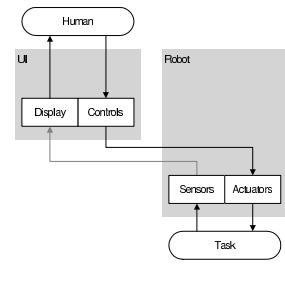

二 efseroratrath

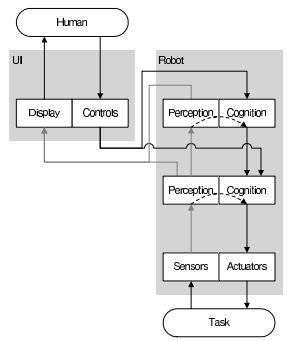

(b)

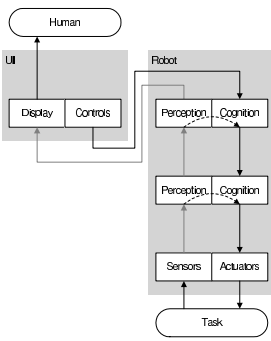

(c)

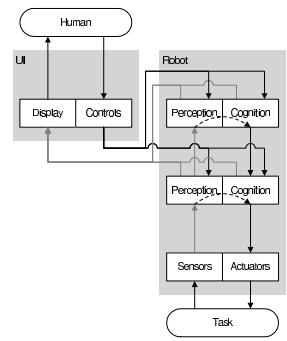

$(d)$

Figure 1. Teleoperation system models: a, direct control; b, supervisory control; c, fully autonomous control; d, collaborative control.

The most common method for vehicle teleoperation is direct control: the operator operates the vehicle using hand-controllers while monitoring video displays (Figure 1a). Because all control decisions depend on the human, system performance is directly linked to human capabilities. Many factors including skill, training, etc., all play a role in how the system functions. Other factors, such as communication bandwidth, may also influence operational efficacy. 
The supervisory control system model is shown in Figure 1b. With supervisory control, the human divides a problem into a sequence of tasks which the robot performs on its own [9]. Once he gives control to the robot, the human typically assumes a monitoring role. However, the human may also intermittently (i.e., trade or share) control the robot by closing a command loop or he may control some variables while leaving the others to the robot.

Fully autonomous control is somewhat of a misnomer because it rarely is fully automatic. With this system model, the human gives high-level goals, which the robot independently achieves (Figure 1c). The difference between supervisory and fully autonomous control is the nature of the goal. In the former, goals are limited and task planning is performed primarily by the human. With the latter, goals are more abstract and the robot is responsible for planning.

With conventional system models, poor performance (or failure) will occur if the human fails to recognize that the robot is ill-suited for the task or situation. Additionally, none of the models can effectively accommodate a wide range of users. Direct control, for example, is generally limited to trained, expert users because difficulty and risk are high.

\section{Collaborative Control System Model}

Collaborative control addresses the limitations of conventional system models through collaboration and dialogue. In supervisory or fully autonomous control, if the robot has difficulties, the only choices it has are to continue performing poorly or to stop. With collaborative control, however, the robot has the option of asking the human to assist: providing information, helping perform perception or cognition, etc.

Another way in which collaborative control differs from conventional system models is that it provides fine-grained sharing/trading of control and autonomy adjustments. Because work is dynamically allocated through dialogue, the human is automatically included in the control loop as needed. This is a significant difference from other models, which require the user to decide how, when, and where control should be allocated.

With collaborative control, therefore, the human may be involved in multiple control loops (Figure 1d). As with supervisory control, he may close a command loop or monitor task execution through interface displays. As a resource for the robot, however, the human may also close a perception loop, a cognition loop, or some combination of the two. Furthermore, the human may interact with the robot at different levels of abstraction. 


\section{System Design}

We have implemented collaborative control as a set of modules (dialogue management, robot control, etc.), each of which operates with variable autonomy in a message-based architecture[4]. Whether a module operates at a low level (strongly dependent on the human) or high level (little or no human interaction) of autonomy is determined by factors including situational demands, module competency, and user model.

In our system, dialogue is the exchange of messages between human and robot. Our current system has approximately thirty messages: human-to-robot (command, queries, responses) and robot-tohuman messages (information statements, queries). At present, the robot is able to ask two types of queries. Safeguard queries concern safety issues (e.g., "Stopped due to rollover danger. Can you come over and help?". Task queries describe task-specific functions, such as "Motion detected. Is this an intruder (image)? If you answer yes, I will follow him."

Each robot query is described by a number of attributes, some of which are operator-dependent (required response accuracy, required expertise) and others which are operator-independent (expiration, priority, etc). These attributes are used to select which queries will be asked. A failure to respond, whether intentional or not, can trigger behavioral changes (e.g., the robot's level of autonomy may be increased).

\section{Results}

\subsection{Remote Driving Tests}

During the past year, we have conducted a variety of remote driving tests to evaluate the use of collaborative control[4,5]. In one test, we examined the use of multiple mobile robots for reconnaissance and surveillance. One of the attractive features of collaborative control is that it directs the human's limited resources (attention, cognition, etc.) where they are needed. This relieves the human of the burden of simultaneously monitoring each robot.

Figure 2 shows an experiment in which an operator used two robots to perform outdoor reconnaissance. During the test, each robot asked safety-related questions while traversing unknown terrain. For example, both robots had questions about setting safety levels. Although it is common practice to define "normal" safety levels, there are some occasions when a system must be used beyond its design specifications. This is particularly true for situations in which system loss is acceptable as long as the goal is achieved (e.g., military combat missions). 

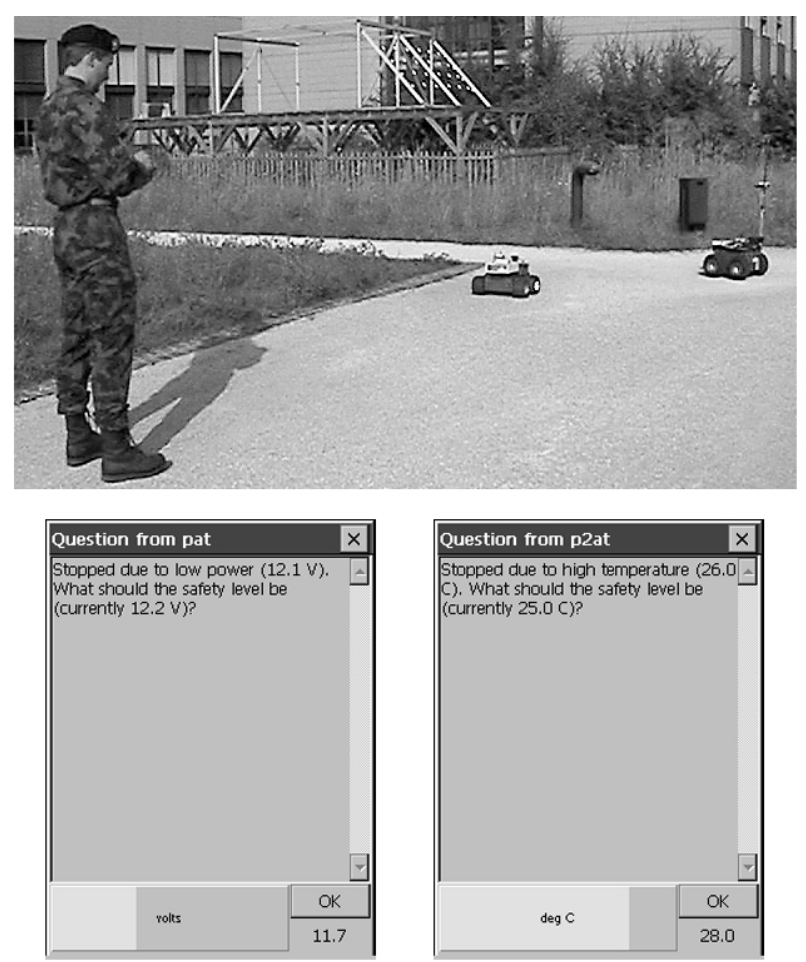

Figure 2. Outdoor reconnaissance with two robots

\subsection{User Study}

To examine how collaborative control influences human-robot interaction, we recently performed a Contextual Inquiry (CI) user study[4]. $\mathrm{CI}$ is a structured interviewing method for grounding interactive system design in the context of the work[6]. In the study, users were required to explore a cluttered environment while assisting safeguarding autonomy.

The study revealed several interesting findings. We observed that: (1) different users may respond quite differently to the same question; (2) users may grow weary of answering questions; (3) a question without adequate detail is hard to answer; (4) dialogue can make users personify the robot; and (5) indicating the urgency of questions is important.

Overall, we found dialogue to be valuable for teleoperation. In particular, novices reported that dialogue significantly helped them understand the problems the robot encountered during task execution. Although experts were generally less satisfied than novices, primarily because they grew tired of answering questions, they also stated that dialogue was a useful in keeping them involved and engaged in system operation. 


\section{Discussion}

\subsection{Benefits of Collaborative Control}

Unlike other forms of teleoperation, in which the division of labor is defined a priori, collaborative control allows human-robot interaction and autonomy to vary as needed. If the robot is capable of handling a task autonomously, it can do so. But, if it cannot, the human can provide assistance.

The use of dialogue makes human-robot interaction adaptable. Since the robot is aware of the user, it can always decide if asking a question

will be useful. Because it has knowledge of the human's expertise, accuracy, etc., the robot can consider whether to accept a response at face value or to weigh it against other factors. In this way, system operation can adapt to different operators.

Dialogue helps the human to be effective. By focusing attention where it is needed, dialogue helps coordinate and direct problem solving. In particular, in situations in which the robot does not know what to do, or when it is working poorly, a simple human answer (even a single bit of information from a novice) is often all that is required to get the robot out of trouble.

\subsection{Limitations of Collaborative Control}

Although collaborative control is beneficial to teleoperation, there are limits to what it can provide. If human-robot interaction is adaptive, then control and information flow will vary with time and situation. This can make validation and verification difficult because it becomes harder to duplicate an error condition or a failure situation.

Another consideration is that when humans and robots interact to achieve common goals, they are subject to team related issues. In particular, teamwork requires team members to coordinate and synchronize their activities, to exchange information and communicate effectively, and to minimize the potential for interference between themselves. Moreover, there are numerous factors which can impact and limit group performance including resource distribution, timing, progress monitoring, and procedure maintenance.

Finally, working in collaboration requires that each partner trust and understand the other. To do this, each collaborator needs to have an accurate model of (1) what the other is capable of doing and (2) how he will carry out a given assignment. If the model is inaccurate, or if the partner cannot be expected to perform correctly, then the collaboration will not work well. 


\section{Open Issues}

As we have discussed, collaborative control provides a framework for coordinating and adapting robot operation. The issue of scaleability, however, remains to be addressed. For example, if the human must interact with a large number of robots, it might not be possible for him to assist each one individually. Instead, it would be more practical to focus on group interaction and have the robots work in formation. Similarly, if a robot has many varied modules, it may be difficult for the human to help different types of autonomy. In this case, it might be more efficient for the human to only assist certain modules.

In more general terms, human assistance is clearly a limited resource. Hence, we need to find ways of motivating the user to respond. This is particularly important when the robot must operate for long periods. One approach would be to develop an algorithm for choosing questions that are "significant" to the user, i.e., having some level of information theoretical content (bits), matching user interest, etc.

\section{Acknowledgments}

This work was partially supported by grants from the DARPA ITO MARS program, the National Science Foundation, and SAIC.

\section{References}

[1] J. Albus et al. NASA/NBS Standard reference model for telerobot control system architecture (NASREM). Technical Note 1235, NIST, 1987.

[2] M. Bauer et al. A Collaborative Wearable System with Remote Sensing. In Proc. of 2nd International Symposium on Wearable Computers, 1998.

[3] G. Dorais et al. Adjustable autonomy for human-centered autonomous systems. In Proc. of Workshop on Adjustable Autonomy Systems (IJCAI), 1999.

[4] T. Fong. Collaborative control: a robot-centric model for vehicle teleoperation. PhD thesis, Carnegie Mellon University, 2001.

[5] T. Fong et al. Multi-robot remote driving with collaborative control. IEEE Transactions on Industrial Electronics (in press), 2002.

[6] K. Holtzblatt and S. Jones. Contextual inquiry: a participatory technique for system design. In D. Schuler and A. Namioka (eds.), Participatory design: principles and practice. Lawrence Erlbaum, 1993.

[7] R. Murphy and E. Rogers. Cooperative assistance for remote robot supervision. Presence, 5(2), 1996.

[8] J. Rosenblatt DAMN: A distributed architecture for mobile navigation. In Proc. of AAAI Spring Symposium on Lessons Learned from Implemented Software Architecture for Physical Agents, 1995.

[9] T. Sheridan. Telerobotics, automation, and human sup. control. MIT Press, 1992. 